\title{
Comparação de diferentes testes de campo para a avaliação da flexibilidade geral ativa
}

\section{Artigo Original}

\author{
Juan Benavent \\ juan.benavent@uv.es \\ Victor Tella' \\ victor.tella@uv.es \\ Ismael González-Millan² \\ ismael.gonzález.millan@unileon.es \\ Juan C. Colado' \\ juan.colado@uv.es
}

\begin{abstract}
Benavent J, Tella V, González-Millan I, Colado JC. Comparação de diferentes testes de campo para a avaliação da flexibiliade geral ativa. Fit Perf J. 2008;7(1):26-9.
\end{abstract}

RESUMO: Introdução: $\bigcirc$ objetivo deste estudo foi identificar um teste de campo válido para avaliação da flexibilidade geral ativa, mediante a comparação de várias provas, tanto tradicionais como mais recentes. Materiais e Métodos: A amostra foi composta por 68 estudantes universitários de ambos os sexos. Os testes empregados foram: flexão profunda do corpo (FP); "sit and reach" (SR); "tot-flex" original (TFO); e "tot-flex" melhorado (TFM). Para determinar o caráter preditor da flexibilidade geral ativa de cada um destes testes, foram relacionadas as medições obtidas em cada um deles com o somatório de 14 medidas angulares goniométricas realizadas de forma ativa ( $\left.\sum M A G A\right)$, pertencentes às principais ações mecânico-articulares de maior amplitude de movimento corporal. Resultados: As correlações entre cada um dos 4 testes analisados e o $\sum M A G A$, foram: $F P=0,53 ; S R=0,53$; $T F O=0,77$; e $T F M=0,77$. Discussão: Em função destes dados, podese concluir que tanto o TFO quanto o TFM são os mais válidos para a avaliação da mobilidade corporal geral ativa, pela sua alta correlação com a mobilidade angular objetiva de referência.

Palavras-chaves: goniometria articular, estiramento muscular dinâmico, tot-flex original, tot-flex modificado.

Endereço para correspondência: Faculty of Sciences of the Physical Activity and of the Sport - Department of Physical Education and Sports - University of Valencia - C/ Gascó Oliag 3, C.P. 46010 - Valencia - España

\begin{tabular}{|l|l|l|}
\hline 7 & 1 & $26-29$ \\
\hline
\end{tabular}


Comparison of different field tests for the evaluation of the general active flexibility

Introduction: The objective of this study was to identify a valid field test for evaluation of the general active flexibility, by the comparison of several races, for traditional and recent ones. Materials and Methods: The sample was composed by 68 academical students of both sexes. The used tests were: deep body flexing (DF); "sit and reach" (SR); original "tot-flex"(OTF); and improved "tot-flex" (ITF). To determine the predicter character of the general active flexibility of each one of these tests, were related the measurements obtained in each one of them with the sum of 14 angular goniometrical measures accomplished in an active way ( $\Sigma M A G A)$ belonging to the main mechanic-articular actions of larger width of corporal movement. Results: The correlations between each one of the 4 analyzed tests and $\sum M A G A$, were: $D F=0.53 ; S R=0.53 ; O T F=0.77$; and ITF $=0.77$. Discussion: In function of these data, it can be ended that OTF and ITF are the most valid for the evaluation of the corporal general active mobility, for its high correlation with the reference angular mobility lens.

Keywords: articular goniometry, dynamic muscular stretching, original tot-flex, modified tot-flex.
Comparación de diferentes tests de campo para la evaluación de la flexibilidad general activa

Introducción: El objetivo de este estudio fue identificar un test de campo valido para la evaluación la flexibilidad general activa mediante la comparación de varias pruebas tanto tradicionales como más recientes. Materiales y Métodos: La muestra fue compuesta de 68 estudiantes universitarios de ambos sexos. Los tests empleados fueron el de flexión profunda del cuerpo (FP), "sit and reach" (SR), "tot-flex" original (TFO) y "tot-flex" mejorado (TFM). Para determinar el carácter predictor de la flexibilidad general activa de cada uno de los tests fueron relacionadas las mediciones obtenidas en cada uno de ellos con la sumatoria de 14 medidas angulares goniométricas realizadas de forma activa ( $2 M A G A)$ pertenecientes a las principales acciones mecánico-articulares de mayor amplitud de movimiento corporal. Resultados: Las correlaciones entre cada uno de los cuatro tests analizados y el $\sum M A G A$ fueron: $F P=0,53 ; S R=0,53$; $T F O=0,77$; y $T F M=0,77$. Discusión: En función de estos datos se puede concluir que tanto el TFO como el TFM son los más válidos para la valoración de la movilidad corporal general activa por su alta correlación con la movilidad angular objetiva de referencia.

Palabras clave: goniometría articular, estiramiento muscular dinámico, tot-flex original, tot-flex modificado.

\section{INTRODUÇÃO}

A flexibilidade músculo-articular é específica de cada articulação, sendo que uma pessoa não pode ser considerada como flexível com base na aplicação de um teste que meça apenas uma articulação. $O$ adequado é que a soma de todas as articulações, ou o maior número delas, determine o valor da flexibilidade, ou que no teste aplicado para tal determinação participem várias articulações ao mesmo tempo e seja validado para tal efeito'. Desde que esta perspectiva cobre sentido dos testes de campo de mobilidade corporal geral, se entendendo como tal aqueles que incidem sobre várias articulações em uma mesma ação motriz ${ }^{2}$, a diferença dos testes de mobilidade específica ou concreta são aqueles que se centram em avaliar só uma articulação ${ }^{3}$. Por sua vez, se entende por mobilidade ativa quando a força de aplicação é produzida pelo próprio indivíduo através da contração muscular dos músculos almejados.

A avaliação da mobilidade corporal geral ativa está evoluindo sensivelmente desde que as técnicas aplicadas para sua avaliação se realizam com medições objetivas, através dos diferentes aparelhos de medição. Existem numerosos testes de campo para valorar a mobilidade corporal geral ativa, mesmo que poucos sejam validados corretamente e aceitos nos diferentes âmbitos da atividade física e do esporte ${ }^{4,5}$. Seguindo as sugestões de González Millán (1997), há a necessidade de comparação de diferentes testes de campo que sejam considerados de mobilidade corporal geral ativa para poder determinar qual deles pode ser o mais adequado por seu maior caráter preditor. Alguns dos mais tradicionais são o de SR de Wells \& Dillon ${ }^{6}$ e o de FP de Litwin \& Fernández ${ }^{7}$. Entre os mais novos, destacam-se o TFO de Porta ${ }^{8}$ e o TFM de González Millán?. Em geral, todos eles apresentam a mobilidade corporal geral (músculo-articular) dos indivíduos de forma ativa, mesmo que sempre se deva levar em conta que os resultados de ditos testes de campo podem estar influenciados pelas medidas antropométricas dos próprios indivíduos avaliados. Para poder inferir que um teste é mais válido que outro, deve-se fazer a medição de numerosos núcleos articulares e com este resultado estabelecer uma correlação com o valor único dos testes ativos gerais que se tenham empregado?. Portanto, ante a falta de estudos científicos que tenham comprovado que testes de mobilidade geral ativa podem ser os mais válidos, o presente trabalho de pesquisa pretendeu identificar os que se aplicam habitualmente na atualidade.

\section{MATERIAIS E MÉTODOS}

\section{Amostra}

Os sujeitos estudados foram 68 adultos, estudantes universitários, de ambos os sexos (34 homens e 34 mulheres), com uma média de idade de $22,6 \pm 2,7$ anos. 
Tabela 1 - Valores médios (m) e desvio padrão (dp) dos testes de campo e da $\sum$ MAGA de referência

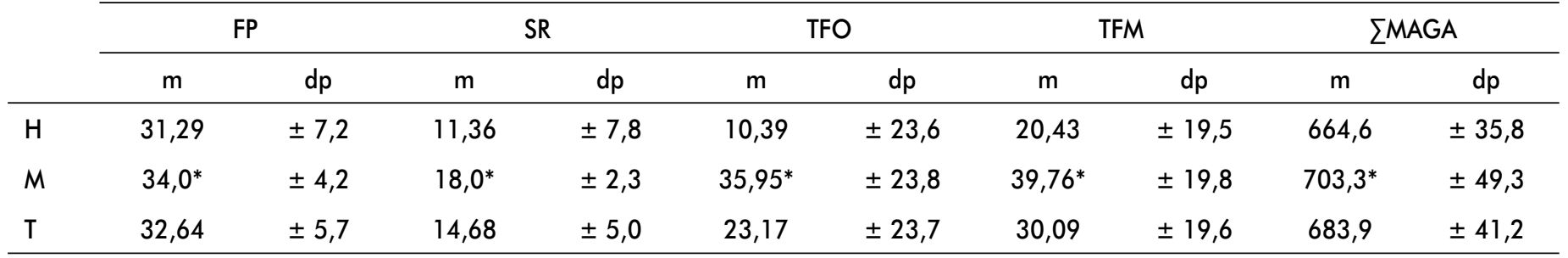

$H=$ homem; $M=$ mulher; $T=$ total

*diferença estatisticamente significativa entre sexos $(p<0,05)$

Tabela 2 - Correlação entre os testes de campo e o IMAGA de referência, com um coeficiente ( $r$ ) estatisticamente significativo de $\mathrm{p}<0,05$

\begin{tabular}{lcccc}
\cline { 2 - 5 } & FP & SR & TFO & TFM \\
\hline Homens & 0,49 & 0,46 & 0,68 & 0,70 \\
Mulheres & 0,53 & 0,43 & 0,71 & 0,71 \\
Total & 0,53 & 0,53 & 0,77 & 0,77 \\
\hline
\end{tabular}

\section{Material}

Utilizou-se: um goniômetro convencional para medir as amplitudes angulares, em graus, das principais ações mecânicoarticulares corporais; uma lança ou barra de madeira redonda, de $3,5 \mathrm{~cm}$ de diâmetro, para a medição de deslocamento de ombros e avaliação das duas versões do tot-flex; um banco sueco de $30 \mathrm{~cm}$ de altura, com uma tábua acoplada retangular de madeira de 30×60×2cm; e uma fita métrica metálica convencional, para medir o deslocamento de ombros e os resultados de todos os testes de campo.

\section{Protocolo de mediçc̃o}

Todas as medições foram realizadas na mesma semana, com uma temperatura ambiental controlada de $22 \pm 0,1^{\circ} \mathrm{C}$. Os sujeitos assistiram a uma sessão de familiarização com as provas de avaliação, 48h antes da primeira sessão de avaliação. A partir desse momento, não realizaram nenhum

\section{Figura 1 - Flexão profunda do corpo (FP)}

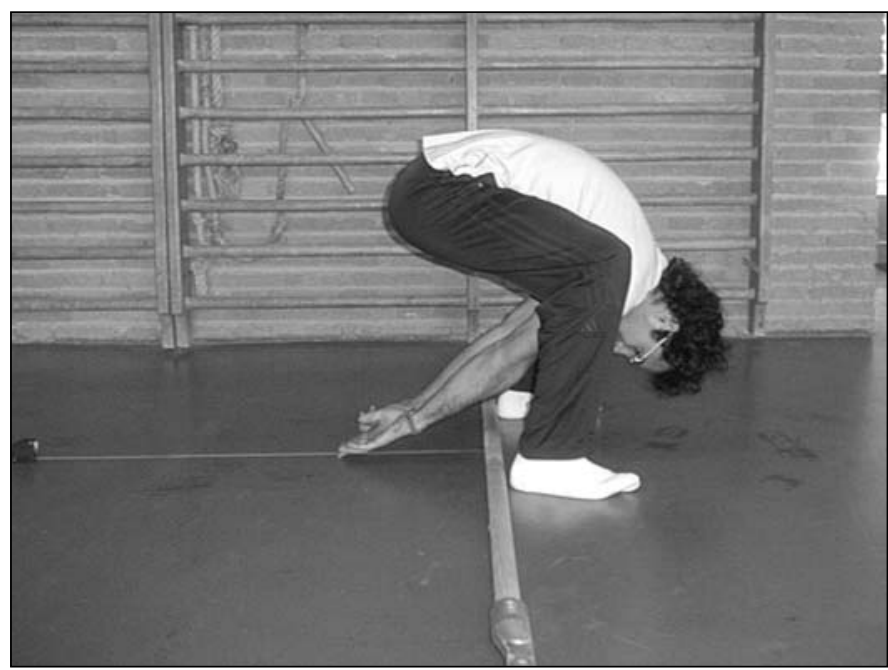

Tabela 3 - Correlação do $\sum$ MAGA de referência, com os $50 \%$ dos sujeitos mais flexíveis e os $\mathbf{5 0 \%}$ dos menos flexíveis, em relação ao TFO e o TFM

\begin{tabular}{lll}
\cline { 2 - 3 } & TFO & TFM \\
\hline $50 \%$ - flexíveis & 0,67 & 0,69 \\
$50 \%+$ flexíveis & 0,80 & 0,79 \\
\hline
\end{tabular}

tipo de esforço físico intenso. A análise da confiabilidade do teste e repetição obtidos apresentaram um alto coeficiente $(R=0,90)$. Todas as medições das provas foram realizadas com o mesmo material, posição e técnicos. Os examinadores estavam adequadamente treinados e qualificados. As indicações que se seguiram para a aplicação das provas foram as habituais $\mathbf{6}^{\mathbf{7}, \mathbf{7}, 9}$.

Todos os sujeitos realizaram um aquecimento padrão antes de proceder à realização dos diferentes testes. As articulações sobre as que se incidiu tal aquecimento foram as dos ombros, punhos, quadris, tornozelos e coluna vertebral. Durante os períodos de pausa entre as diferentes medições, os sujeitos realizaram suaves movimentos ativos para manter os efeitos provocados pelo aquecimento. As medições angulares com o goniômetro avaliaram as 14 ações mecânico-articulares analíticas indicadas: (i) flexão plantar de tornozelo; (ii) flexão dorsal de tornozelo; (iii) flexão de quadril com joelho estendido; (iv) extensão de quadril com joelho fletido; (v) adução de quadril;

\section{Figura 2 - Sit and reach com banco (SR)}

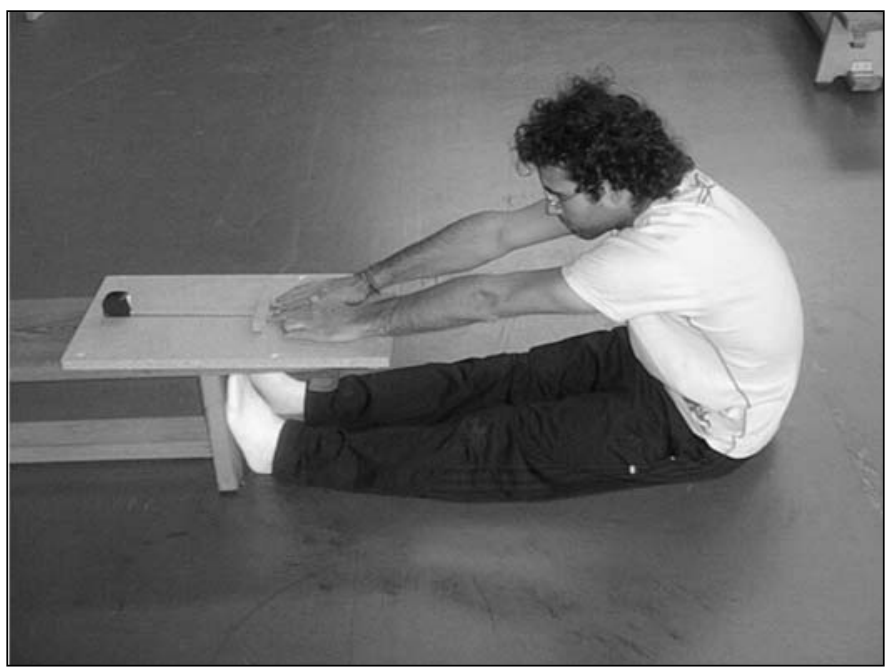




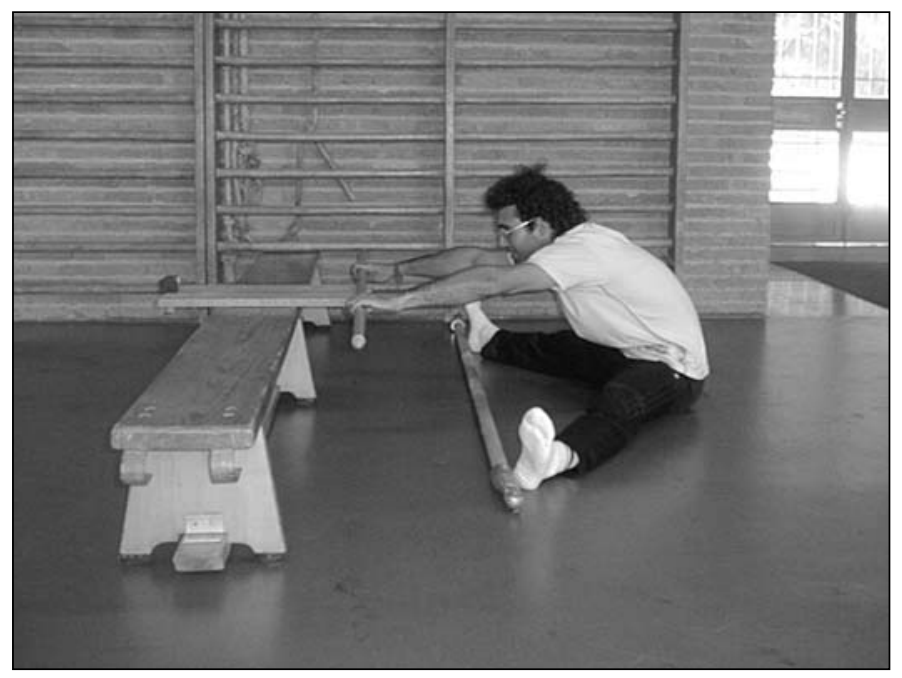

(vi) abdução de quadril; (vii) flexão de coluna torácico-lombar; (viii) extensão de coluna torácico-lombar; (ix) abdução de ombro; (x) flexão horizontal de ombro; (xi) flexão de ombro; (xii) extensão de ombro; (xiii) flexão de punho; e (xiv) extensão de punho. Além disso, se realizou a prova de "deslocamento de ombros" como parte inicial e característica do teste tot-flex. A seguir, procedeu-se a realização, de maneira padronizada, dos testes globais de campo, na seguinte ordem: DB (Figura 1), SR (Figura 2), TFO (Figura 3) e TFM (Figura 4). Em cada prova permitiu-se 2 ensaios prévios e, a seguir, realizaram-se 2 medições, levando sempre a melhor marca para a análise final. Entre cada ensaio ou prova, recuperou-se 30s.

\section{Método de comparação e análise estatística}

Uma vez realizada a análise descritiva habitual, realizou-se um estudo comparativo, de correlação linear simples, entre os valores médios de cada teste de campo de medição linear indireta da mobilidade e o valor padrão ou somatório das 14 medições diretas angulares de mobilidade músculo-articular. Posteriormente, foi realizado este estudo comparativo de correlação entre o $\Sigma M A G A$ de referência e os 2 testes de campo mais destacados. Neste caso, dividindo-os em 2 metades, segundo os melhores e piores resultados de cada um desses testes, respectivamente. $O$ pacote estatístico empregado foi o SPSS - V 11.5 para Windows.

\section{RESULTADOS}

Os valores médios, com os respectivos desvios típicos de cada teste, estão refletidos na Tabela 1. As correlações de cada teste de campo com o $\Sigma$ MAGA de referência se mostram na Tabela 2. Já que, tanto o TFO como o TFM, são as 2 provas de campo com maior correlação com o $\sum$ MAGA, na Tabela 3 se mostram suas correlações diferenciando os sujeitos por $50 \%$ de melhores e piores resultados sem diferenças de sexos.
Figura 4 - Tot-flex modificado com banco (TFM)

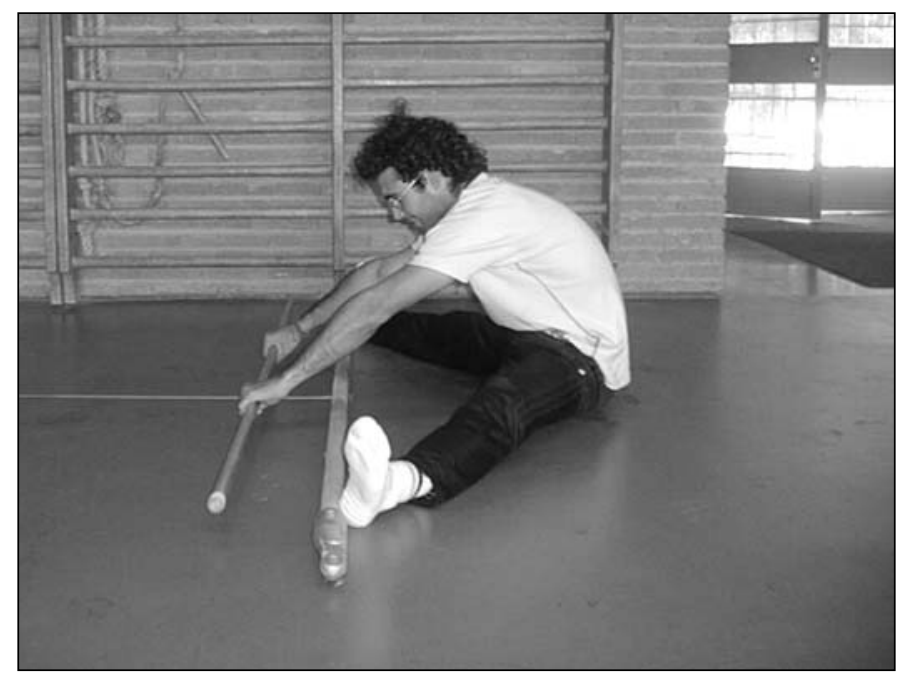

\section{Discussão}

Os resultados mostrados na Tabela 2 sugerem uma correlação média dos testes de FP e SR com o $\sum$ MAGA. Também mostram que as duas versões de tot-flex possuem uma alta correlação com - LMAGA. Estes dados podem sugerir a validade de qualquer tipo de versão de tot-flex na avaliação da mobilidade corporal geral ativa, muito acima dos outros 2 testes que podem ser mais conhecidos ou populares. Inclusive, na Tabela 3, se aprecia como qualquer versão do top-flex é mais preditiva nos sujeitos mais flexíveis do que nos menos flexíveis. Finalmente, ressalte-se que na Tabela 1 se aprecia a existência de uma diferença estatisticamente significativa entre os valores obtidos por gênero em todos os testes, sendo sempre mais favorável para as mulheres, ratificando-se assim o fato conhecido de que as mulheres são mais flexíveis que os homens.

\section{REFERÊNCIAS BIBLIOGRÁFICAS}

1. Corbin C, Noble L. Flexibility: a major component of physical fitness. Journal of Physical Education and Recreation. 1980;51:57-60.

2. García JM, Navarro M, Ruíz JA. Pruebas para la valoración de la capacidad motriz en el deporte. Madrid: Gymnos;1995.

3. Weineck J. Importancia y entrenamiento de la flexibilidad. REEFD 1989;21:9-21.

4. Moras G. Anàlisi crítica dels actuals tests de flexibilitat. Correlació entre alguns dels tes ts actuals i diverses mesures antropomètriques. Apunts: Educación Física Deportiva. $1992 ; 24: 127-37$

5. Rodríguez FA, Aragonés MT. Valoración funcional de la capacidad de rendimiento físico. En: Gonzalez J. Fisiología de la actividad física y del deporte. Madrid: McGrawHill; 1992.

6. Wells K, Dillon E. The sit and reach: a test of back and leg flexibility. Research Quaterly. 1952;23:115-8.

7. Litwin J, Fernández G. Evaluación y estadísticas aplicadas a la Educación física y el Deporte. Buenos Aires: Stadium; 1974.

8. Porta J. El desarrollo de las capacidades físicas: la flexibilidad. Apunts: Educación Física y Deportiva. 1987;7:10-9.

9. González-Millán I. Validación de pruebas de campo para la medición de la flexibilidad y su relación con la estructura corporal [tese]. León: Servicio de Publicaciones de Fisiología; 1997. 